\title{
The Neglected (Abdominal) Compartment: What Is New at the Beginning of the 21st Century?
}

\author{
Zsolt J. Balogh · Ari Leppäniemi
}

Published online: 12 April 2009

(C) Société Internationale de Chirurgie 2009

Perfusion of the organs in any body cavity or compartment can be compromised when intracompartmental pressure exceeds pressure in the capillaries. This simple pathophysiological principle is well recognized in many areas of the body and often presents in life- or limb-threatening scenarios. The detrimental consequences of increased intracranial pressure, tension pneumothorax, pericardial tamponade, and extremity compartment syndromes have been known for at least a century. The existence and significance of these syndromes are rarely questioned.

Interestingly, the abdominal compartment in this regard was neglected for a long time. Although several studies during the 19th century recognized the consequences of increased intraabdominal pressure (IAP), this knowledge never reached the physiology books and was largely unknown to the surgical society. Around the mid-20th century the problem was re-recognized by pediatric surgeons during attempts to close large omphaloceles. Later, starting with surgery for abdominal aortic aneurysms and damage control surgery for trauma, many surgical subspecialties described the lethal combination of increased IAP and the development of acute organ failure. The term abdominal compartment syndrome (ACS) was coined to describe this newly re-described syndrome, which responded to timely decompression with marked improvement in organ function.

\section{Z. J. Balogh ( $₫)$}

Trauma Service, Department of Surgery, John Hunter Hospital,

University of Newcastle, University Drive, Callaghan,

NSW 2308, Australia

e-mail: Zsolt.Balogh@hnehealth.nsw.gov.au

\section{A. Leppäniemi}

Department of Surgery, Meilahti Hospital, University

of Helsinki, PO Box 340, Helsinki, HUS 00029, Finland
During the last 15 years, our understanding of ACS went through the usual pathway of characterizing a newly reported syndrome in modern medical history. ACS was first reported by case reports as well as seminal reviews and expert opinions. This stage was followed by consistent work of specialty groups to characterize the syndrome, which had various etiologies: It was seen in patients who experienced trauma, those with pancreatitis, mixed intensive care unit patients, emergency general surgical patients, and patients with severe burns.

More recently, researchers who have focused interest on this topic combined their efforts under the aegis of the World Society of Abdominal Compartment Syndrome (WSACS) to develop consensus definitions, foster research in identified key areas, and achieve better outcomes of this lethal syndrome through education, prevention, and evidence-based treatment strategies.

The following review articles from experts in their fields summarize our current knowledge and identify further directions for research on different forms of ACS. This symposium was endorsed by the WSACS and the International Association for Trauma Surgery and Intensive Care (IATSIC), and it complements these societies' biannual meetings in June 2009 in Dublin (WSACS) and September 2009 in Adelaide (IATSIC). 